\title{
Consequences of a possible jet-star interaction in the inner central parsec of Centaurus $A$
}

\author{
C. Müller ${ }^{* a, b}$, M. Kadler ${ }^{a}$, K. Mannheim ${ }^{a}$, M. Perucho ${ }^{c, d}$, R. Ojha ${ }^{f}$, E. Ros ${ }^{e, c, d}$, \\ R. Schulz ${ }^{a, b}$, J. Wilms ${ }^{b}$ \\ ${ }^{a}$ Institut für Theoretische Physik und Astrophysik, Universität Würzburg, Germany \\ ${ }^{b}$ Dr. Remeis Observatory \& ECAP, 96049 Bamberg, Germany \\ ${ }^{c}$ Observatori Astronòmic, Universitat de València, Paterna, València, Spain \\ ${ }^{d}$ Departament d'Astronomia i Astrofisica, Universitat de València, Burjassot, València, Spain \\ ${ }^{e}$ Max-Planck-Institut für Radioastronomie, Auf dem Hügel 69, 53121 Bonn, Germany \\ ${ }^{f}$ NASA Goddard Space Flight Center, USA \\ E-mail: cornelia.muellerdastro.uni-wuerzburg.de
}

\begin{abstract}
The jet-counterjet system of the closest radio-loud active galaxy Centaurus A (Cen A) can be studied with Very Long Baseline Interferometry (VLBI) on unprecedented small linear scales of $\sim 0.018 \mathrm{pc}$. These high-resolution observations provide essential information on jet emission and propagation within the inner parsec of an AGN jet. We present the results of a kinematic study performed within the framework of the Southern-hemisphere AGN monitoring program TANAMI. Over 3.5 years, the evolution of the central-parsec jet structure of Cen A was monitored with VLBI. These observations reveal complex jet dynamics which are well explained by a spine-sheath structure supported by the downstream acceleration occurring where the jet becomes optically thin. Both moving and stationary jet features are tracked. A persistent local minimum in surface brightness suggests the presence of an obstacle interrupting the jet flow, which can be explained by the interaction of the jet with a star at a distance of $\sim 0.4 \mathrm{pc}$ from the central black hole. We briefly discuss possible implications of such an interaction regarding the expected neutrino and high-energy emission and the effect on a putative planet.
\end{abstract}

12th European VLBI Network Symposium and Users Meeting,

7-10 October 2014

Cagliari, Italy

\footnotetext{
* Speaker.
} 


\section{Introduction}

At a distance of 3.8 Mpc [1], Centaurus A (Cen A) is the closest radio-loud active galactic nucleus (AGN) exhibiting powerful radio jets. Thanks to its proximity it is one of the most interesting targets to address open questions of AGN jet physics. Very Long Baseline Interferometry (VLBI) enables us to study the jet at sub-parsec scales [2,3]. It is detected up to $\gamma$-ray energies showing a double-humped broadband spectrum $[4,5]$. Cen A is a potential candidate for ultra-high energy cosmic ray (UHECR) emission [6,7] and furthermore it coincides positionally with the highest energy PeV neutrino event detected by IceCube [8]. Detailed investigations of Cen A can give a better understanding of jet formation and emission mechanisms in extragalactic jets in general.

\section{Results from TANAMI monitoring of Centaurus A}

Figure 1 shows the first seven TANAMI (Tracking Active Galactic Nuclei with Austral Milliarcsecond Interferometry, [9]) VLBI epochs at $8.4 \mathrm{GHz}$ of Cen A [3] using Southern telescopes in Antarctica, Australia, Chile, South Africa, and New Zealand. With sub-milliarcsecond (mas) resolution we can study the innermost part of Cen A's jet in great detail, resolving individual features at linear scales of $\sim 0.018 \mathrm{pc}$ (corresponding to 1 mas at $3.8 \mathrm{Mpc}$ ). The TANAMI monitoring reveals complex jet dynamics in the inner parsec. We detect a position-dependent acceleration downstream of the jet. The region with higher apparent component speeds coincides with the part where the jet becomes optically thin [2] and can hence be explained with a spine-sheath structure. We found a range of speeds of $0.1 c$ to $0.3 c$. Using the ratio of the jet-to-counterjet brightness, the jet angle to the line of sight can be constrained to $\theta \sim 12^{\circ}-45^{\circ}$. This result is consistent with findings by Hardcastle et al. [10] for the kpc-scale jet, while only our lower limit overlaps with previous VLBI measurements by Tingay et al. [11] at $\sim(3-15)$ mas resolution. The monitoring also shows long-term stable features. The second brightest component at $\sim 3.5$ mas next to the core is found to be stationary with almost constant brightness temperature. It can be identified with the stationary component at $\sim 4$ mas found by [12] at lower angular resolution. It has a steep spectrum [2] and can be explained as a jet nozzle locally enhancing the internal pressure. Farther downstream, at $\sim 25$ mas from the core, the jet shows a widening and the surface brightness decreases, resembling a "tuning fork" (see Fig. 1). This persistent local minimum in surface brightness suggests the presence of an obstacle interrupting the jet flow. Detailed considerations (see [3]) lead to a possible interpretation as the interaction of the jet with the stellar wind of a red giant.

\section{Jet-star interaction}

When interpreting the "tuning fork" observed in the inner parsec of Cen A's jet as the interaction with a red giant, further possible consequences of such an event can be considered. In the following we point these out and briefly discuss some implications.

\subsection{Production of $\gamma$-rays}

From theoretical calculations and simulations (e.g., $[13,14,15,16])$ we can expect $\gamma$-ray production from the interaction of an AGN jet with a massive object like a star, though those 
emission events are on short timescales (hours to days). However, assuming multiple interactions within the jet ${ }^{1}$, the persistent $\gamma$-ray emission observed from Cen A [4] could be partly produced by such processes. Non-thermal particles are generated in the bow shock between the jet and the stellar wind, leading to synchrotron and inverse Compton emission. Potential $\gamma$-ray emission from a single jet-star interaction is the highest for objects close $(\sim 1 \mathrm{pc})$ to the jet base [13]. According to the calculations by Araudo et al. [13], one star interacting at a distance of $\sim 1 \mathrm{pc}$ with a jet with a luminosity of $\sim 10^{43-44} \mathrm{erg} \mathrm{s}^{-1}$ produces a $\gamma$-ray luminosity of $\sim 10^{37-38} \mathrm{erg} \mathrm{s}^{-1}$. These values are comparable to what we can expect from the scenario in Cen A. This emission is likely to be outshined by the mildly beamed $\gamma$-ray emission of Cen A $\left(\sim 10^{41} \mathrm{erg} \mathrm{s}^{-1}\right.$, [17]), although such misaligned sources are potential candidates to be detected at high energies owing to such an event [13]. Furthermore, Barkov et al. [16] argue that a very high energy (VHE) flare from jet-star interactions could be detectable, though not ruling out that such interactions might also contribute to the persistent VHE emission in Cen A [5].

Intriguingly, the analysis of four years of Fermi/LAT $\gamma$-ray data of the Cen A core emission reveals evidence for a second, harder power law component above $\sim 4 \mathrm{GeV}$ [18] which could be due to an additional emission mechanism besides the jet-related synchrotron emission.

\subsection{Production of neutrinos}

Prompted by the IceCube detection of "Big Bird", one of the three PeV neutrino events [8], which is positionally consistent with Cen A, we estimate the neutrino events from a jet-star interaction in Cen A. The interaction of protons accelerated at a bow shock with radius $r_{\mathrm{BS}}=3 \times 10^{16} \mathrm{~cm}$ surrounding a red giant and its wind can also lead to the emission of high-energy neutrinos resulting from the hadronic production of pions. In an optically thick target, this mechanism turns about half of the energy carried by relativistic protons and ions into neutrinos, and the other half into $\gamma$-rays [19]. Assuming a red supergiant with a very large radius $r_{\mathrm{RG}}=8 \mathrm{AU}$ such as UY Scuti, we thus obtain a maximum neutrino luminosity of

$$
L_{v}=\frac{1}{2} L_{p p \rightarrow \pi}=\frac{1}{2} L_{\mathrm{p}}\left(\frac{r_{\mathrm{RG}}}{r_{\mathrm{BS}}}\right)^{2}=\frac{1}{2}\left(\frac{r_{\mathrm{BS}}}{r_{\mathrm{jet}}}\right)^{2} L_{\mathrm{jet}}\left(\frac{r_{\mathrm{RG}}}{r_{\mathrm{BS}}}\right)^{2} \approx 5 \times 10^{36} \mathrm{erg} \mathrm{s}^{-1}
$$

adopting $L_{\text {jet }}=6.5 \times 10^{43} \mathrm{erg} \mathrm{s}^{-1}$ [4]. Although the degenerate core of such a star is optically thick also to the high-energy neutrinos, most of its cross sectional area is still transparent.

Additional neutrino flux is generated by the optically thin comet-tail-like wind with $\tau_{\mathrm{pp} \rightarrow \pi}=$ $\left(n_{\mathrm{p}} / 10^{6} \mathrm{~cm}^{-3}\right) \sigma_{\mathrm{pp} \rightarrow \pi}\left(r_{\mathrm{w}} / 200 \mathrm{AU}\right) \approx 1.4 \times 10^{-4}$. The assumed density of $10^{6} \mathrm{~cm}^{-3}$ corresponds to the expected value for a cylindrical expansion of the wind from the stellar surface with $n_{\mathrm{p}}=$ $10^{9} \mathrm{~cm}^{-3}$ out to $200 \mathrm{AU}$. The corresponding neutrino luminosity is given by

$$
L_{v}=\frac{1}{2} \tau_{\mathrm{pp} \rightarrow \pi} L_{\mathrm{p}}\left(\frac{r_{\mathrm{w}}}{r_{\mathrm{BS}}}\right)^{2}=\frac{1}{2} \tau_{\mathrm{pp} \rightarrow \pi} L_{\mathrm{jet}}\left(\frac{r_{\mathrm{BS}}}{r_{\mathrm{jet}}}\right)^{2}\left(\frac{r_{\mathrm{w}}}{r_{\mathrm{BS}}}\right)^{2} \approx 5 \times 10^{35} \mathrm{erg} \mathrm{s}^{-1}
$$

The Larmor radius of protons $r_{\mathrm{L}} \approx 10^{13}(B / 6 \mathrm{G})^{-1}\left(\gamma_{\mathrm{p}} / 2 \times 10^{7}\right) \mathrm{cm}$ is small enough to permit Fermi acceleration up to multiple PeV energies at the bow shock. Due to the quasi-stationary target medium and shock, relativistic beaming does not increase the apparent flux, unless the accelerated

\footnotetext{
${ }^{1}$ Note, that the radio and X-ray knots in the kpc-scale jet could be explained by interaction of stars with the jet [10].
} 
particle distribution is different from the one predicted by the Fermi mechanism (e.g., beam-like). Assuming the above neutrino luminosity is concentrated at $\mathrm{PeV}$ energies, the expected number of contained PeV neutrino events during three years of IceCube data taking reaches a value of only $\sim 10^{-5}$, rendering an association of the Big Bird neutrino event with a neutrino from a single jet-star interaction with a red giant highly improbable.

\subsection{What would happen to an Earth-like planet?}

In addition to the TANAMI results, the study by Hardcastle et al. [10] also suggests the presence of multiple jet-star interactions in Cen A. It can be assumed that the stars are at different evolution stages. The case of a sun-like star orbited by an Earth-like planet gives rise to the question of how such a planet would be affected by the interaction. According to our calculations in [3] the interaction of Cen A's jet and a sun-like star with a stellar wind of $v_{W}=400 \mathrm{~km} \mathrm{~s}^{-1}$ and a mass loss rate of $\dot{M}=2.5 \times 10^{-14} \mathrm{M}_{\odot} \mathrm{yr}^{-1}$ results in a distance to the contact discontinuity of $\sim 7$ AU. Therefore, an Earth-like planet would still "be safe" behind the contact discontinuity, i.e., not affected by dynamical effects. For a higher Lorentz factor, the contact discontinuity can reach 1 AU. However, we caution that a more detailed statement on the propagation of the shock front and how it affects the planet requires more sophisticated simulations taking the planet's atmosphere and surface conditions into account.

Nevertheless, a lot more life threatening is the $\gamma$-ray emission to which the planet would be exposed. Strong $\gamma$-ray radiation can destroy a putative, protective ozone layer in an Earth-like planet with harmful consequences for life because of the subsequent increase of UV radiation reaching the surface (see e.g., [20, 21]). Thomas et al. [21] performed detailed calculations of the influence of $\gamma$-ray bursts (GRBs, with typical luminosities of $\sim 10^{51} \mathrm{erg} \mathrm{s}^{-1}$ ) on the Earth's atmosphere. According to them, a $\gamma$-ray fluence of $100 \mathrm{~kJ} \mathrm{~m}^{-2}$ (burst duration $10 \mathrm{~s}$ ) would cause significant damage to life due to $-38 \%$ ozone depletion (global average). For comparison, $10 \mathrm{~kJ} \mathrm{~m}^{-2}(-16 \%$ depletion) would have a smaller effect and $1000 \mathrm{~kJ} \mathrm{~m}^{-2}$ (up to $-80 \%$ local depletion) will result in a total damage. The authors argue that the ionizing X-ray fluence from the burst afterglow could cause further ozone depletion and a significant amount of X-ray radiation would arrive at ground without the protective ozone layer. Naively adopting the considerations for GRBs by Thomas et al. [21] and Piran \& Jiménez [22] we can make simple estimations on the expected $\gamma$-ray fluence at the position of the planet while it is exposed to the emission from Cen A's jet and compare the result with GRBs. Taking Cen A's core $\gamma$-ray luminosity of the order of $\sim 10^{41} \mathrm{erg} \mathrm{s}^{-1}$ [4] and the distance to the AGN core of a few parsecs ${ }^{2}$, the ozone layer will be depleted within minutes to a few hours. This duration is substantially reduced when considering beamed emission. To stay below the critical fluence value of $\sim 100 \mathrm{~kJ} \mathrm{~m}^{-2}$ the system needs to be at least at a distance of $\mathscr{O}(200 \mathrm{pc})$ from the AGN core for a jet crossing time of about $\sim 20$ years. These estimations strongly depend among other things on the inclination of the system and the orbital parameters of the planet, e.g., the time for which a close planet is protected from the $\gamma$-ray radiation by its host star. Considering other types, from hot or cold rocky Mercury-like to Jupiter- or Neptune-like gaseous, planets, the impact of such a star-jet interaction further strongly depends on their magnetic field, the composition of their atmosphere, and the stellar wind.

\footnotetext{
${ }^{2}$ For this simple estimate at these scales the lower $\gamma$-ray flux density from the kpc-scale lobes [23] can be neglected.
} 


\section{Conclusion}

We presented our recent results from the Southern-Hemisphere VLBI monitoring of Cen A which reveal complex jet kinematics in the inner parsec. A long-term stable feature, the "tuning fork", can be interpreted as the interaction of the jet with a star of the host galaxy. We point out several possible consequences of such a scenario which require further follow-up analysis and simulations to test, e.g., whether there exists a high-energy emission component in the Cen A spectrum from jet-star interactions.

\section{Acknowledgments}

C.M. and R.S. wish to thank RadioNet3 for support to attend this meeting. C.M. acknowledges the support of the Bundesministerium für Wirtschaft und Technologie (BMWi) through Deutsches Zentrum für Luft- und Raumfahrt (DLR) grant 50 OR 1404. E.R. was partially supported by the Spanish MINECO project AYA2012-38491-C02-01 and by the Generalitat Valenciana project PROMETEOII/2014/057. M.P. is a member of the work team of projects AYA2013-40979-P and AYA2013-48226-C3-2-P, funded by the Spanish Ministerio de Economìa y Competitividad (MINECO), and PROMETEOII/2014/069, funded by Generalitat Valenciana. We acknowledge the COST MP0905 action 'Black Holes in a Violent Universe'.

\section{References}

[1] Harris G.L.H., Rejkuba M., Harris W.E., 2010, Proc. Astron. Soc. Aust. 27, 457

[2] Müller C., Kadler M., Ojha R., et al., 2011, A\&A 530, L11

[3] Müller C., Kadler M., Ojha R., et al. 2014, A\&A 569, 115

[4] Abdo A.A., Ackermann M., Ajello M., et al., 2010, ApJ 719, 1433

[5] Aharonian, F., Akhperjanian, A. G., Anton, G., et al. 2009, ApJL 695, L40

[6] Kim, H. B. 2013, ApJ 764, 121

[7] Pierre Auger Collaboration, Abraham, J., Abreu, P., et al. 2007, Science 318, 938

[8] Aartsen, M. G., Ackermann, M., Adams, J., et al. 2014, Physical Review Letters, 113, 101101

[9] Ojha R., Kadler M., Böck M., et al., 2010, A\&A 519, A45

[10] Hardcastle M.J., Worrall D.M., Kraft R.P., et al., 2003, ApJ 593, 169

[11] Tingay S.J., Jauncey D.L., Reynolds J.E., et al., 1998, AJ 115, 960

[12] Tingay S.J., Preston R.A., Jauncey D.L., 2001, AJ 122, 1697

[13] Araudo A.T., Bosch-Ramon V., Romero G.E., 2013, MNRAS 436, 3626

[14] Khangulyan D.V., Barkov M.V., Bosch-Ramon V., et al., 2013, ApJ 774, 113

[15] Bosch-Ramon V., Perucho M., Barkov M.V., 2012, A\&A 539, A69

[16] Barkov M.V., Aharonian F.A., Bosch-Ramon V., 2010, ApJ 724, 1517

[17] Abdo A. A., Ackermann M., Ajello M., et al. 2010, ApJ 720, 912

[18] Sahakyan N., Yang R., Aharonian F.A., Rieger F.M., 2013, ApJ 770, L6 


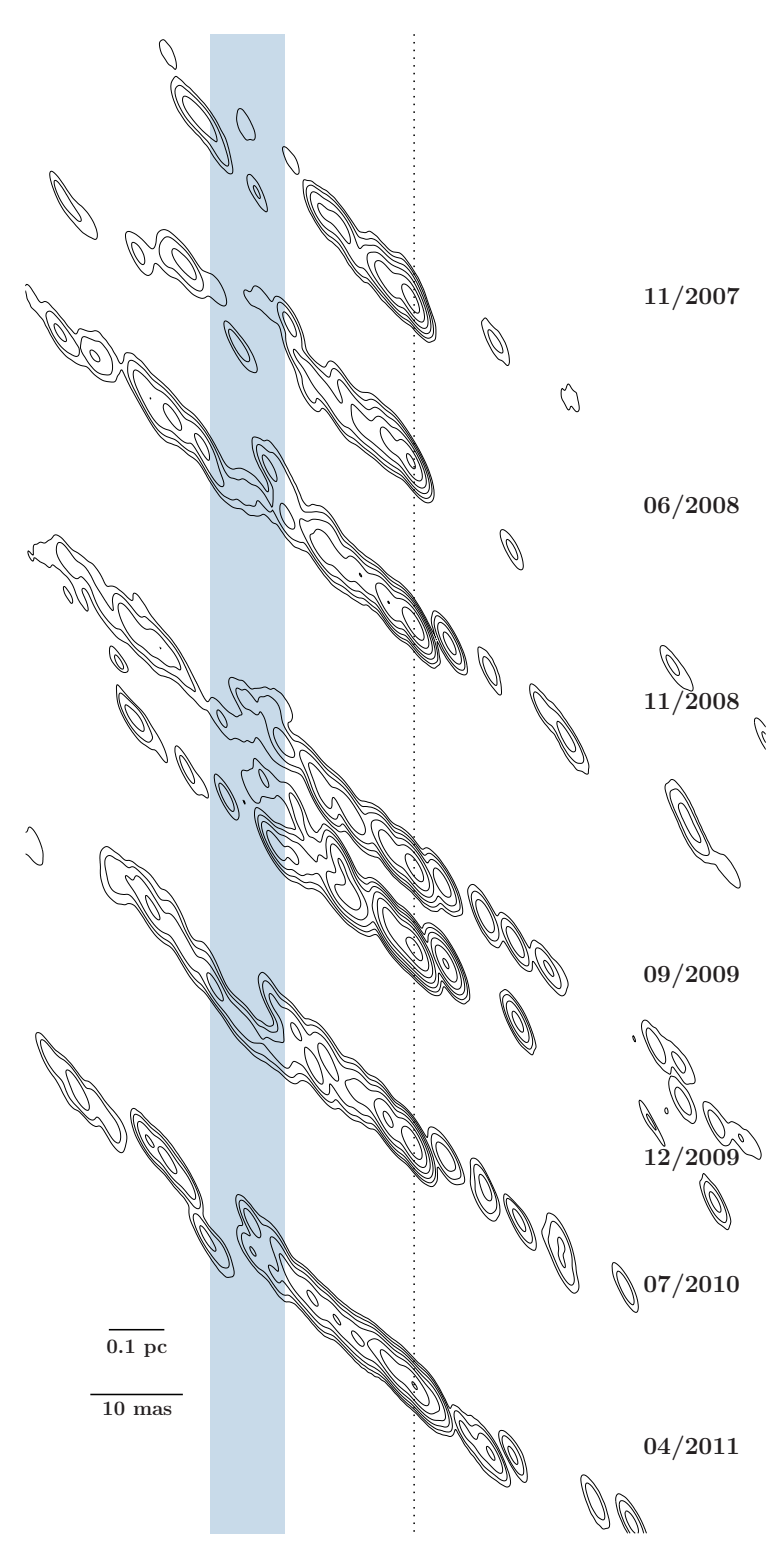

Figure 1: Results from TANAMI monitoring. Shown is the time evolution of the inner parsec of Cen A's jet at $8.4 \mathrm{GHz}$. Contour clean images, restored with a common beam of $(3.33 \times 0.78)$ mas at P.A. $=26.3^{\circ}$, the phase center is indicated by the dashed vertical line. The contours indicate the flux density level, scaled logarithmically and increased by a factor of 3 , with the lowest level set to the $5 \sigma$-noise-level. The blue shaded area at $\sim 25$ mas away from the phase center highlights the "tuning-fork" where the jet widens and the surface brightness decreases (see [3] for details). This feature can be explained by a jet-star interaction.

[19] Mannheim, K., Schlickeiser, R., 1994, A\&A 286, 983

[20] Ruderman M. A. 1974, Science 184, 1079

[21] Thomas B. C., Melott A. L., Jackman C. H., et al. 2005, ApJ 634, 509

[22] Piran T., \& Jiménez R. 2014, Physical Review Letters 113, 231102

[23] Abdo, A. A., Ackermann, M., Ajello, M., et al. 2010, Science, 328, 725 